\title{
Laser-Driven Undulator Radiation
}

\author{
G. Shvets and N. J. Fisch \\ Princeton Plasma Physics Laboratory, Princeton University, Princeton, NJ 08543
}

A. Pukhov

Max Plank Institute for Quantum Optics, Garching-bei-Munchen, Germany

\begin{abstract}
An electromagnetic wake of infra-red radiation can be generated by an intense laser pulse, propagating through an underdense plasma in the presence of a magnetostatic undulator. As opposed to undulator radiation from a charged bunch propagating in a periodic magnetic field, here radiation comes from almost stationary plasma electrons, ponderomotively pushed by the laser pulse. Such laser-driven undulator radiation can be produced by either a single ultra-short pulse, or by two frequency-detuned long pulses. In the latter case the difference frequency is efficiently produced by quasi phase-matched optical heterodyning.
\end{abstract}

\section{INTRODUCTION AND MOTIVATION}

This work is motivated by a search for the new ways of generating high-power tunable far infra-red (FIR) radiation, which is an important research tool in various scientific applications. One such application is to use the FIR radiation as a seed for a free-electron laser (FEL) amplifier. The initially uniform electron beam can emerge from the amplifier, tightly bunched at the right frequency for injecting it into a plasma beatwave accelerator [1]. If the FIR radiation is generated by the same laser beatwave which drives the accelerating plasma beatwave, the beam bunching is naturally synchronized with the plasma beatwave.

We describe a novel Laser-driven Undulator Radiation in the Infrared (LURI), which is produced when a short laser pulse (or laser beatwave) propagates in a plasma-filled undulator. In contrast with the electrostatic wake at $\omega_{p}$ in unmagnetized plasma, or Cerenkov radiation at a frequency close to $\omega_{p}$ in uniformly magnetized plasma [2], the frequency of the LURI radiation is given by $\omega_{s}=\omega_{p}^{2} / 2 k_{w} c$, where $\omega_{p}=\sqrt{4 \pi n e^{2} / m}$ is the plasma frequency, $\lambda_{w}=2 \pi / k_{w}$ is the undulator periodicity, $n$ is the plasma density, $-e$ and $m$ are electron charge and mass, respectively. 


\section{DISCLAIMER}

This report was prepared as an account of work sponsored by an agency of the United States Government. Neither the United States Government nor any agency thereof, nor any of their employees, make any warranty, express or implied, or assumes any legal liability or responsibility for the accuracy, completeness, or usefulness of any information, apparatus, product, or process disclosed, or represents that its use would not infringe privately owned rights. Reference herein to any specific commercial product, process, or service by trade name, trademark, manufacturer, or otherwise does not necessarily constitute or imply its endorsement, recommendation, or favoring by the United States Government or any agency thereof. The views and opinions of authors expressed herein do not necessarily state or reflect those of the United States Government or any agency thereof. 


\section{DISCLAIMER}

Portions of this document may be illegible in electronic image products. Images are produced from the best available original document. 
Conventionally, one thinks of undulator radiation as being produced by a relativistic electron beam. Magnetostatic field is Doppler-upshifted into the infrared photons, with the amount of upshift determined by the electron energy, providing the tunability. In a LURI, however, the electron beam is substituted by the stationary plasma and laser pulse. Plasma is the radiating medium, and the laser pulse provides the Doppler shift. The schematic $c$ of the LURI source, recently described by us [3], is shown in Fig. 1. Plasma electrons experience a ponderomotive kick from the laser, giving them axial momentum. The undulating magnetic field couples with the longitudinal motion of the electrons to produce transverse acceleration, causing them to radiate in the axial direction.

As in undulator radiation, a "radiation zone", roughly defined as the extent of the laser pulse, propagates with relativistic speed in the direction of the undulator periodicity. Here, however, the Doppler upshift is caused by the group velocity of the laser pulse instead of the, typically small, axial velocity of the plasma electrons. LURI is emitted by mostly stationary (although transversely accelerated) plasma electrons.

In this paper we present a detailed treatment of LURI, expanding upon earlier one-dimensional calculation [3](Section II). While the original calculation emphasized the LURI excitation by an ultra-short pulse, here we emphasize an alternative excitation method by a beatwave of two long, suitably detuned in frequency, laser pulses (Section III). This method of producing the difference frequency radiation is compared with the Cerenkov source (Section IV). In Section III we also present the results of the two-dimensional Particle-in-Cell (PIC) simulation of the beatwave excitation of the LURI and discuss the applications of this radiation scheme to beam injection into a plasma beatwave accelerator.

\section{GENERAL DESCRIPTION}

We start with simple 1-D geometry, assuming that the laser is infinite in $x-y$ plane. Linearly polarized undulator $\vec{B}_{w}=B_{w} \cos \left(k_{w} z\right) \vec{e}_{x}$ laser pulse $\vec{A}_{0}=\vec{e}_{x} a_{0}(\zeta) m c^{2} / e \cos \left(\omega_{0} t-k_{0} z\right)$ are assumed, where $\zeta=v_{g} t-z$, and $\omega_{0} \gg \omega_{p}$,

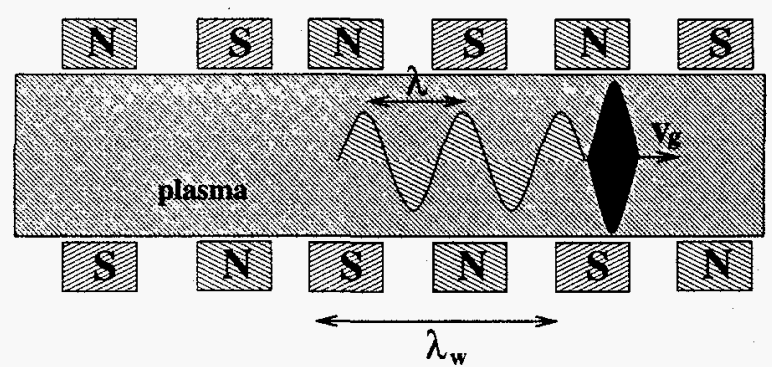

FIGURE 1. Schematic for generation of a laser-driven undulator radiation. The resonant wavelength $\lambda=2 \lambda_{p}^{2} / \lambda_{w}$. 
so that $v_{g} \approx c$. Plasma electrons experience several forces: ponderomotive force $\vec{F}_{p}=\vec{e}_{z} m c^{2} \partial\left(a_{0}^{2} / 2\right) \partial \zeta$, restoring force of the fixed ions, $\vec{v} \times \vec{B}_{w}$ force of an undulator, and the electric fields of the LURI. Linearized equations of motion are

$$
\begin{aligned}
& \ddot{\xi}_{z}=-\omega_{p}^{2} \xi_{z}-\omega_{B} \cos \left(k_{w} z\right) \dot{\xi}_{y}+\frac{c^{2}}{4} \frac{\partial a_{0}^{2}}{\partial \zeta} \\
& \ddot{\xi}_{y}=\omega_{B} \cos \left(k_{w} z\right) \dot{\xi}_{z}-e E_{s} / m
\end{aligned}
$$

where $\xi_{y}, \xi_{z}$ are Lagrangian electron displacements, $\omega_{B}=e B_{w} / m c, E_{s}$ is the LURI electric field, and $\dot{\xi}=\partial \xi / \partial \zeta$.

We further assume that (a) LURI is significantly above the plasma cutoff (a true electromagnetic mode!) $\omega_{s} \gg \omega_{p}$, and (b) plasma is weakly magnetized $\omega_{B} \ll \omega_{s}$. Hence, all forces except the ponderomotive can be neglected in the RHS of the longitudinal equation of motion (1), yielding $\dot{\xi}_{z}=c a_{0}^{2}(\zeta) / 4$. Therefore, only the electrons overlapping with the laser pulse are moving axially, thereby experiencing a $z$-dependent $\vec{v} \times \vec{B}_{w}$ undulator acceleration. Thus, periodic magnetic field has two functions: (i) enabling plasma electrons to radiate in the forward direction by imparting a finite transverse acceleration $\dot{v}_{y}$; and, (ii) ensuring constructive interference of the radiation emitted from different regions along the undulator. The combination of a laser pulse, moving at a speed approaching $c$, and a periodic magnetic field introduces the necessary spatio-temporal correlation between different regions of the plasma, resulting in a Doppler-upshifted radiation.

In 1-D condition (ii) is equivalent to requiring that the phase of the LURI changes by $-2 \pi$ as the laser pulse moves by one wiggler period: $k_{s z} \Delta z-\omega_{s} \Delta t=-2 \pi$, where $\Delta z=\lambda_{w}$ and $\Delta t=\lambda_{w} / v_{g}$. This can be recast in a form familiar from FEL physics, where the group velocity of the laser $v_{g}=c \sqrt{1-\omega_{p}^{2} / \omega_{0}^{2}}$ assumes the role of the beam velocity:

$$
\left(k_{s z}+k_{w}\right) v_{g}=\omega_{s}
$$

where $\omega_{s}$ and $k_{s z}$ are related through the dispersion relation in unmagnetized plasma $\omega_{s}^{2}=k_{s z}^{2} c^{2}+\omega_{p}^{2}$. Equation (3) is only meaningful when the frequency of the LURI $\omega_{s}$ is much smaller than the frequency of the driver laser $\omega_{0}$.

Equation (3) determines the frequency of the emitted radiation, and, depending on $\omega_{0}, \omega_{p}$, and $k_{w}$, can have two or no solutions. The two solutions coalesce when $\omega_{p}^{2} / c^{2}=k_{w} k_{0}$. For $\omega_{p}<k_{w} k_{0}$ two solutions exist: $\omega_{s}<\omega_{0}$ and $\omega_{U}>\omega_{0}$. The most interesting case is

$$
k_{w} \ll \omega_{p} / c \ll \sqrt{k_{0} k_{w}}
$$

when the two solutions are, approximately, given by

$$
\omega_{s}=\frac{\omega_{p}^{2}+k_{w}^{2} c^{2}}{2 k_{w} c} \quad \text { and } \quad \omega_{U}=2 k_{w} c \omega_{0}^{2} / \omega_{p}^{2}
$$


When condition (4) is satisfied, it turns out that $\omega_{s} \ll \omega_{0} \ll \omega_{U}$. For example, for $n_{0}=4.0 \cdot 10^{15} \mathrm{~cm}^{-3}, \lambda_{w}=1 \mathrm{~cm}, \lambda_{0}=1.0 \mu \mathrm{m}$ we find $\lambda_{s}=50 \mu \mathrm{m}, \lambda_{U}=200 \AA$. Another important distinction between the low-frequency and high-frequency solutions is that the former is almost independent of the group velocity of the laser (as long as $v_{g} \approx c$ ), while the latter is proportional to $\gamma_{g}^{2}$, where $\gamma_{g}=1 / \sqrt{1-v_{g}^{2} / c^{2}}$ is the relativistic factor of the laser pulse. The high-frequency solution is more reminiscent of the undulator radiation from a relativistic beam, upshifted by $\gamma_{\|}^{2}$. As we explain below, the rate of emission at $\lambda_{U}$ is negligible. However, by appropriately shaping the laser pulse, or by utilizing a beatwave of two long frequency-detuned lasers, a considerable amount of far infra-red/ $\mathrm{THz}$ radiation at $\lambda_{s}$ can be obtained.

Geometry of the problem dictates that the electric and magnetic fields of the LURI point in $y$ in $x$ directions, respectively. Neglecting the end effects near the entrance and exit of an undulator, the electric field of the LURI is assumed as $\vec{E}=\vec{e}_{y} E_{y}(\zeta) \exp \left(-i k_{w} z\right) / 2+$ c. c. We substitute this expression into the wave equation for the transverse electric field $\left(-c^{2} \nabla^{2}+\partial^{2} / \partial t^{2}\right) E_{y}=4 \pi e n_{0} \ddot{\zeta}_{y}$, where $\ddot{\zeta}_{y}$ is given by Eq. (2). After some algebra, obtain

$$
-i \frac{v_{g}}{\omega_{s}+\omega_{U}}\left(\frac{\partial}{\partial \zeta}+i \frac{\omega_{s}}{v_{g}}\right)\left(\frac{\partial}{\partial \zeta}+i \frac{\omega_{U}}{v_{g}}\right) E_{y}=i \frac{k_{p}^{2}}{8 k_{w}} B_{w} a_{0}^{2}(\zeta)
$$

Equation (6) is integrated, yielding $E_{y}=E_{\omega_{s}}+E_{\omega_{U}}$, where

$$
E_{\omega_{s}}=i \frac{k_{p}^{2} B_{w}}{8 k_{w}} \frac{\omega_{s}+\omega_{U}}{\omega_{U}-\omega_{s}} e^{-i \omega_{s} \zeta / c} \int_{-\infty}^{\zeta} d \zeta^{\prime} e^{i \omega_{s} \zeta^{\prime} / c} a_{0}^{2}\left(\zeta^{\prime}\right)
$$

and

$$
E_{\omega_{U}}=i \frac{k_{p}^{2} B_{w}}{8 k_{w}} \frac{\omega_{s}+\omega_{U}}{\omega_{s}-\omega_{U}} e^{-i \omega_{U} \zeta / c} \int_{-\infty}^{\zeta} d \zeta^{\prime} e^{i \omega_{U} \zeta^{\prime} / c} a_{0}^{2}\left(\zeta^{\prime}\right)
$$

Equations $(7,8)$ indicate that a finite duration laser pulse leaves behind a wake of electromagnetic radiation which contains the two resonant frequencies $\omega_{s}$ and $\omega_{U}$, and the amplitude of each of the frequency components is proportional to the Fourier transform of the laser intensity profile. When Eq. (4) is satisfied, $\omega_{U} \gg \omega_{0}$, and the Fourier transform of the time-averaged laser intensity, evaluated at $\omega_{U}$, vanishes. Therefore, there is no laser-driven undulator radiation at the higher frequency, upshifted by $\gamma_{g}^{2}$, and the frequency of the LURI is equal to $\omega_{s}$.

Mathematically, Eq. (6) has two solutions with different frequencies because it is a second order differential equation. Since the high-frequency contribution is negligible, Eq. (6) can be turned into a first-order equation by noting that $\partial / \partial \zeta \approx$ $-i \omega_{s} / c$ and $\omega_{U} \gg \omega_{s}$ :

$$
\left(\frac{\partial}{\partial \zeta}+i \frac{\omega_{s}}{c}\right) E_{y}=i \frac{\omega_{s}}{4 c} B_{w} a_{0}^{2}(\zeta)
$$


The solution to Eq. (9) is given by $E_{\omega_{s}}$. It was was studied in Ref. [3] with an emphasis on the LURI excitation by a "matched pulse"flat-top laser pulse of duration $\tau_{L}=\pi / \omega_{s}$, resulting in $\left|E_{y}\right|=a_{0}^{2} B_{w} / 2$. The emphasis of this paper is on the laser beatwave excitation of the LURI. We briefly note that a wake of stationary periodic magnetic field is left behind a laser pulse, as demonstrated earlier [3]. This wake vanishes in the regime of the beatwave excitation, and will not be discussed in this paper.

\section{BEATWAVE EXCITATION OF LURI: QUASI-MATCHED OPTICAL HETERODYNE}

The matched pulse excitation of the LURI invokes an analogy with the plasma wake generation in unmagnetized plasma. This analogy suggests an alternative approach: to use the beat of two lasers with frequencies $\omega_{1}$ and $\omega_{2}$, suitably detuned by $\omega_{1}-\omega_{2}=\omega_{s}$, to resonantly excite the LURI. This makes the LURI a promising method of difference-frequency generation.

The periodically magnetized plasma is, therefore, a quasi phase-matched optical heterodyne, where the plasma is the nonlinear medium, and the periodicity of the magnetic field creates the quasi-phase matching of the beatwave and the LURI. Using the quantum mechanical language, the high-frequency photon 1 scatters into a lower-frequency photon 2 and a LURI photon.' By energy conservation, $\omega_{s}=\omega_{1}-\omega_{2}$, and by the conservation of the quasi-momentum $k_{s}=k_{1}-k_{2}-k_{w}$. Combining these two conservation laws and assuming that $v_{g} \approx\left(\omega_{1}-\omega_{2}\right) /\left(k_{1}-k_{2}\right)$ yields Eq. (3). This is another way of deriving the frequency of the LURI, which demonstrates the phase-matching role of the undulator - it takes the excess momentum out of the beatwave driven LURI photon, enabling the resonant excitation. Thus, the generation of the LURI is a process of downshifting the high-frequency photons in a periodically magnetized plasma.

To describe the beatwave excitation of the LURI assume two overlapping Gaussian pulses:

$$
\vec{A}_{1,2}=\vec{e}_{x} a_{1,2} m c^{2} / e \cdot \cos \left(\omega_{1,2} t-k_{1,2} z\right) e^{-\zeta^{2} / 2 \tau_{L}^{2}} .
$$

Assuming $\omega_{1}-\omega_{2}=\omega_{s}$, we replace $a_{0}^{2} / 2$ by $a_{1} a_{2} \cos \left(\omega_{s} \zeta / c\right)$ in Eq. (7) to obtain:

$$
|E|_{y}^{\max }=\frac{\pi^{1 / 2} \omega_{s} \tau_{L}}{4} a_{1} a_{2} B_{w}
$$

It is instructive to understand why the LURI amplitude grows linearly with the pulse length. physical mechanism behind this dependence. Consider a plasma electron at a given location $z=\mathcal{N} \lambda_{w}$ along the undulator, executing the steadystate longitudinal oscillation in the electromagnetic beatwave (EMBW) of the two lasers and continuously radiating the LURI. After the second beat of the EMBW this radiation combines in phase with the radiation emitted by an electron at the location $z=(\mathcal{N}-1) \lambda_{w}$ after the first beat of the EMBW. This is because the LURI 
falls behind the driving laser by one wavelength per wiggler period. Thus combined contributions to the LURI travel forward and, once again, add in phase with the radiation emitted after the third beat by an electron at the location $z=(\mathcal{N}+1) \lambda_{w}$, and so on.

In a resonantly driven LURI we observe a phenomenon of constructive interference of the radiation emitted from different places along the undulator. The resonant excitation of the LURI is, thus, of an entirely different origin than, for example, the resonant plasma-wave excitation [4]. The latter results in an increasingly vigorous motion of the plasma electrons and saturates as soon as the wave breaking limit is reached, when the particle displacement becomes comparable with the wavelength of the plasma wave. Increasingly relativistic motion of the plasma electrons is also responsible for the saturation of the plasma beatwave [4]. To the contrary, individual electrons in the LURI source are not resonantly driven; they execute a steady-state longitudinal oscillation in the EMBW with frequency $\omega_{s}$ and amplitude $v_{z} / c=a_{1} a_{2} / 2$.

We simulated the beatwave excitation of the LURI using a 2-D Particle in Cell (PIC) simulation. Since both the LURI and the excitation pulse are, typically, shorter than the undulator length, we've chosen a time-saving "moving window" simulation, where only the plasma electrons inside a moving simulation box are followed. In Fig. 2 the LURI with $\lambda_{s}=3 \lambda_{1}$ is driven by the beatwave of the $\lambda_{1}$ and $\lambda_{2}=1.5 \lambda_{1}$ lasers. The plasma wavelength $\lambda_{p}=10 \lambda_{1}$ is assumed, and the undulator period, corresponding to the exact one-dimensional phase-matching, is $\lambda_{w}=56 \lambda_{1}$. The transverse profiles of the laser pulses in both simulations were Gaussian, with $\sigma=14 \lambda_{1}$, and the width of the simulation box is $200 \lambda_{1}$.

To distinguish between the much more intense laser radiation and the much weaker LURI we chose the laser electric field and the undulator magnetic field to be linearly polarized in $z$ direction. The laser magnetic field is then in $y$ direction, and we can plot the magnetic field of the LURI (which points in $z$ direction) without interference from the laser magnetic field. The undulator $B_{w}=10^{-3} \mathrm{mc} \omega_{1} / e$, and the two lasers have identical normalized vector potentials $a_{1}=a_{2}=0.1$, durations $\tau_{L}=18 \lambda_{1}$, and spotsizes $\sigma=14 \lambda_{1}$. For $\lambda_{1}=10 \mu$ the undulator and laser parameters correspond to $B_{w}=1$ Tesla, $P_{1}=83 \mathrm{GW}$, and $P_{2}=37 \mathrm{GW}$. The peak power of the LURI is then about $100 \mathrm{~kW}$.

One application of the difference frequency generation is beam injection into a plasma beat wave accelerator $[1,5]$. Efficient operation of a plasma beatwave accelerator requires an injector which supplies an electron beam consisting of microbunches separated by a plasma wave. Each of the microbunches should be significantly shorter than the inter-bunch distance to ensure that all the particles are injected into a plasma wave at, roughly, the same accelerating phase. A typical plasma wavelength $\lambda_{p}=100 \mu \mathrm{m}$ [1] requires $\tau_{b}=40 \mathrm{fs}$ long electron bunches (arbitrarily, $\left.c \tau_{b} / \lambda_{p}=1 / 8\right)$ - too short for the modern photoinjectors. Moreover, the injected electrons have to be synchronized with the EMBW of the two lasers.

Since free-electron lasers (FELs) are known to produce a highly bunched electron beam [5], one possibility is to use a high-gain single-pass FEL, injected with a seed 
electromagnetic wave to pre-bunch the beam before injecting it into a plasma beat wave. The challenge is to produce the electromagnetic seed which is synchronized with the electromagnetic beat wave (and, therefore, with the plasma beat wave). The LURI, generated by the electromagnetic beatwave, could be used as a seed. Below we present an examples of a multi-kilowatt $\lambda_{s}=100 \mu \mathrm{m}$ LURI source driven by a beatwave of two $\mathrm{CO}_{2}$ laser pulses with $\lambda_{1}=9.6 \mu \mathrm{m}$ and $\lambda_{2}=10.6 \mu \mathrm{m}$.

We'll choose the pulse durations $\tau_{L}$ and the laser radii $\sigma$ using the following qualitative argument. Peak power of the LURI is maximized when the laser pulse duration is at least $N_{w}$ beats long, so that $c \tau_{L}=N_{w} \lambda_{s}$. For the one-dimensional

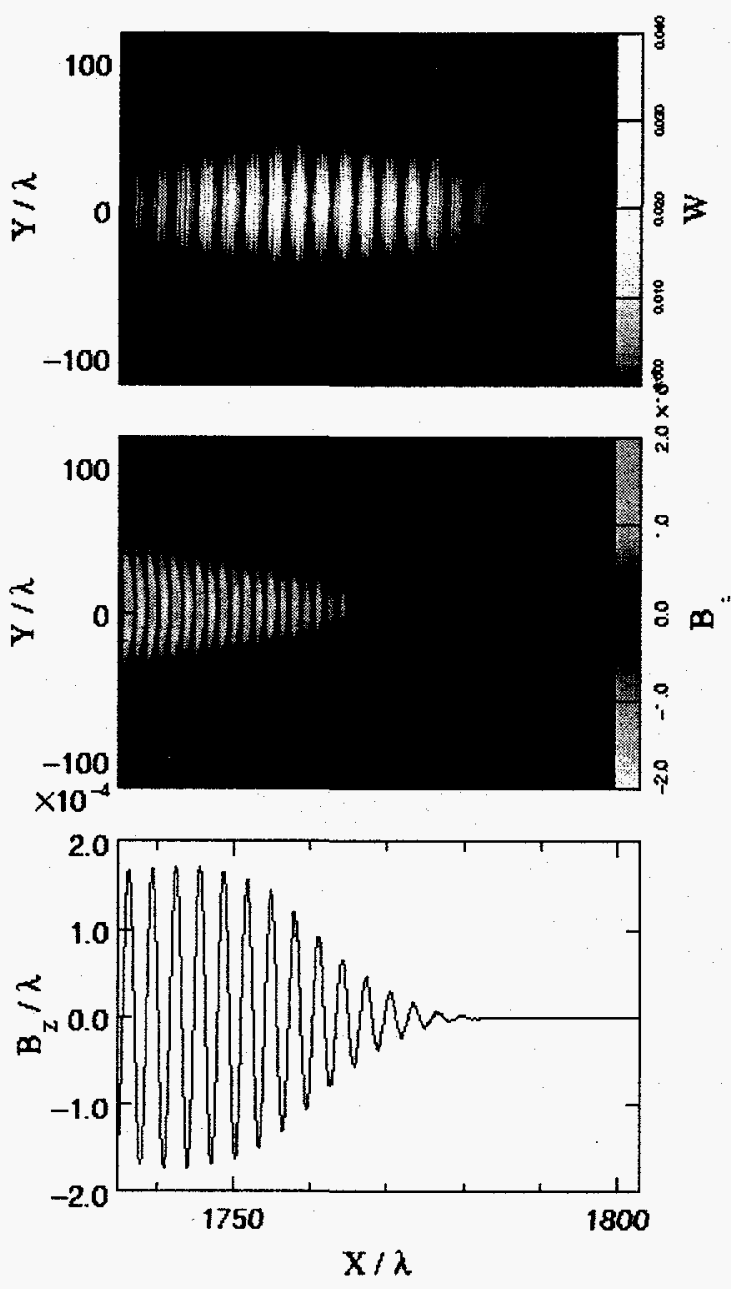

FIGURE 2. Particle in Cell simulation of the beatwave LURI excitation. Laser parameters: $a_{1}=a_{2}=0.1, \sigma=14 \lambda_{1}, \tau_{L}=18 \lambda_{1}$; plasma: $\lambda_{p}=10 \lambda_{1}$; undulator: $\lambda_{w}=56 \lambda_{1}$. (a)-(c) are the snapshots, at $t=1000 \lambda_{1} / c$, of (a) excitation pulses; (b) magnetic field of the LURI (short wavelength $\lambda_{s}=3 \lambda_{1}$ ) and Cerenkov wake (long wavelength $\lambda_{p}$ ), normalized to $m c \omega_{1} / e$; (c) same as (b), on axis. 
theory to remain valid, the LURI has to propagate without diffraction over the entire length of the undulator, so that $N_{w} \lambda_{w}=2 \pi \sigma^{2} / \lambda_{s}$. The peak power of the LURI is then given by

$$
P_{s}=\frac{N_{w} P_{1} P_{2}}{4 P_{0}} \frac{\omega_{B}^{2} \lambda_{1}^{2} \lambda_{2}^{2}}{c^{2} \lambda_{w} \lambda_{s}}
$$

where, for this example, $\lambda_{s}=100 \mu \mathrm{m}$ and $\lambda_{0} \approx 10 \mu \mathrm{m}$.

Using the laser and undulator parameters similar to the ones available at the UCLA Saturnus laboratory [1], we assume $P_{1}=P_{2}=400 \mathrm{GW}, B_{w}=1$ Tesla, $\lambda_{w}=1.5 \mathrm{~cm}$, and $N_{w}=38$. This requires plasma density of $n_{p}=1.3 \times 10^{15} \mathrm{~cm}^{-3}$, laser spotsize $\sigma=1 \mathrm{~mm}$, and $\tau_{L}=15 \mathrm{ps}$. From Eq. (11), $P_{s} \approx 400 \mathrm{kWatt}$ of 100 micron is generated, which, according to Ref. [1], is more than sufficient to inject an FEL amplifier.

\section{COMPARISON OF THE LURI AND CERENKOV WAKES}

An alternative method of the difference-frequency generation by exciting a Cerenkov wake in a uniformly magnetized plasma was recently suggested [2]. Since the two methods are somewhat similar in that they involve magnetized plasma, in this Section we compare the underlying principles of the two approaches.

An important distinction between the resonant excitations of the CW and the LURI is that the former involves phase-matching between the vacuum beatwave and the CW, while the latter involves the quasi phase-matching. The Cerenkov wake (CW) is an extraordinary plasma mode in a uniformly magnetized plasma, which has the frequency $\omega^{(c h)} \approx \omega_{p}$. The exact phase-matching between the beatwave and the CW, $\omega^{(c h)}=\omega_{1}-\omega_{2}$ and $k^{(c h)}=k_{1}-k_{2}$, is achieved in magnetized plasma. Note that such a 3 -wave phase-matching is impossible in unmagnetized plasma. It is the presence of the static magnetic field, which strongly modifies the propagation of the low-frequency Cerenkov wave without significantly affecting the higher frequency drive lasers, that makes phase-matching possible.

The electric field of the CW has both the transverse $\left(E_{y}\right)$ and the longitudinal $\left(E_{z}\right)$ components, connected by the relationship $E_{y} / E_{z}=e B_{0} / m c \omega_{p}$. For example, when a 1 Tesla magnetic field is used to generate 100 micron radiation, the longitudinal field is 100 times larger than the transverse field. Therefore, if high power output (large $E_{y}$ ) is needed, the longitudinal field $E_{z}$ is going to be even larger. This implies large-amplitude longitudinal oscillations of the plasma. Nonlinear effects, such as the relativistic detuning and, possibly, the wave breaking, are likely to become important.

In contrast, the dispersion relation of the LURI is that of a transverse electromagnetic wave since $\omega_{s} \gg \omega_{p}$. Quasi phase-matching is made possible by the periodic undulator field, which modifies the resonance condition by absorbing momentum $k_{w}$ : $\omega^{s}=\omega_{1}-\omega_{2}$ and $k^{s}=k_{1}-k_{2}-k_{w}$. Resonantly driven LURI builds up to 
large power not because of the excitation of the large-amplitude plasma oscillations, but, instead, because of the constructive interference between the radiation emitted at different locations along the plasma. Therefore, plasma nonlinearities will not affect generation of the LURI.

The group velocity of the $\mathrm{CW}$ is $v_{c h} / c=\left(e B_{0} / m c \omega_{p}\right)^{2} \ll 1$, so that there is a substantial group velocity mismatch between the vacuum and the plasma. As was pointed out by the authors [2], a very sharp vacuum/plasma interface (of order $\left.c / \omega_{p}\right)$ is needed to overcome the radiation absorption at the exit from the plasma. Small group velocity can also be viewed as an advantage: the $L_{c h}=c^{2} T_{c h} / v_{c h}$ long plasma region is required to produce a radiation pulse of duration $T_{c h}$. In contrast, a much longer plasma region $L=c T_{c h} \lambda_{w} / \lambda_{s}$ is needed to produce a LURI pulse of the same duration.

Interestingly enough, for identical flat-top laser drivers and magnetic field magnitudes the amplitudes of the CW and the LURI are identical: $E_{y}^{c h}=$ $\left(\omega_{c h} \tau_{L} / 4\right) a_{1} a_{2} B_{0}$ for the CW and $E_{y}^{s}=\left(\omega_{c h} \tau_{L} / 4\right) a_{1} a_{2} B_{w}$ for the LURI. The important difference, however, is that the longitudinal velocity of the plasma electrons in the Cerenkov wake is much larger than in the LURI source: neglecting the relativistic mass increase, $v_{z} / c=a_{1} a_{2}\left(\omega_{p} \tau / 4\right)$.

When relativistic mass increase is taken into account, the peak electron velocity is $v_{z}^{m} / c=\left(16 a_{1} a_{2} / 3\right)^{1 / 3} \quad[4]$, corresponding to the peak transverse field $E_{y}^{(c h)}=$ $v_{z}^{m} B_{0} / c$. For example, for $a_{1}=a_{2}=0.2$ we find $E_{y}^{(c h)}=0.6 B_{0}$. The same LURI intensity is obtained by using identical laser pulses of only $10 \lambda_{s}$ long. Since there is no relativistic detuning, longer pulses result in higher power. The peak intensity stops increasing when the number of beats in the pulse exceeds the number of the undulator periods: $\omega_{s} \tau_{L}>2 \pi N_{w}$. Hence, the limiting peak amplitude of the LURI, generated by the EMBW, is $E_{y}=\pi N_{w} a_{1} a_{2} B_{w} / 2$.

Interestingly, the Cerenkov wake is present even in a periodically magnetized plasma. The undulator field with $k_{w} \ll \omega_{p} / c$ is perceived by the plasma electrons as an almost homogeneous field. Hence, the usual plasma wake, electrostatic in the absence of the external magnetic field, turns into a Cerenkov wake. The magnitude of the wake is slowly modulated with periodicity $\lambda_{w}$. This wake, however, does not escape from the plasma - the nodes of the magnetic field serve as bottlenecks for the field energy since the group velocity vanishes when magnetic field goes to zero. Although the Cerenkov wake appears as a necessary bi-product whenever the LURI is excited, its magnitude can be controlled by adjusting the duration of the laser pulse. Beatwaves tuned to $\omega_{s}$, which are many plasma periods long, do not excite the CW while exciting the LURI. This is because, for a given plasma density, the LURI has a much higher frequency than the CW.

\section{CONCLUSIONS}

In conclusion, a new method for generating coherent tunable radiation in the plasma is described. Radiation is emitted when an laser pulse propagates through 
the plasma in the presence of a static periodic magnetic field. The resulting laserdriven undulator radiation in the infrared (LURI) can be viewed as an electromagnetic wake, propagating along with the pulse, and can be efficiently excited by two methods: using an ultra-short laser pulse, which matches in duration the period of the LURI, or using a long beatwave of two lasers, detuned by the LURI frequency. The latter excitation scenario was numerically modeled using direct Particle-in-Cell (PIC) simulations. The beatwave generation of the LURI is, to our knowledge, the first example of a quasi phase-matched optical difference frequency generation of high-intensity FIR radiation in the plasma. This easily tunable radiation can be used for injecting a free-electron laser with the purpose of pre-bunching an electron beam. Since the beam bunching would be naturally synchronized with the laser beatwave, this scheme may be used as an injector into a plasma beatwave accelerator. Comparisons between this and an alternative method of difference-frequency generation, the beatwave excitation of the Cerenkov wake, are also presented.

\section{ACKNOWLEDGMENTS}

This work was supported by the United States Department of Energy, Division of High-Energy Physics. We gratefully acknowledge discussions with Dr. T. Katsouleas.

\section{REFERENCES}

1. M. Lampel, C. Pellegrini, R. Zhang, C. Joshi, and W. M. Fawley, Proceedings of the 1995 Particle Accelerator Conference, v. 2, 764 (IEEE, New York, NY, 1995).

2. J. Yoshii, C. H. Lai, T. Katsouleas, C. Joshi, and W. B. Mori, Phys. Rev. Lett. 79, 4194 (1997).

3. G. Shvets, N. J. Fisch, and J.-M. Rax, Phys. Rev. Lett. 80, (1998).

4. M. N. Rosenbluth and C. S. Liu, Phys. Rev. Lett. 29, 701 (1972).

5. C. E. Clayton and L. Serafini, IEEE Trans. Plasma Sci. 24, 400 (1996). 